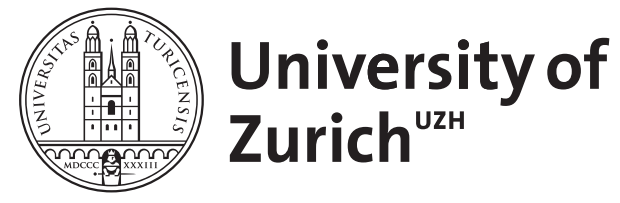

\title{
Impfung gegen Pneumokokken überzeugt nicht restlos
}

Djalali, S ; Badertscher, N

\begin{abstract}
Die Pneumokokkenimpfung gehört nicht zu den «beliebtesten» Impfungen. Bis vor kurzem empfahl das Bundesamt für Gesundheit, Risikopatienten jeden Alters und Menschen über 64 Jahre generell zu impfen. Dennoch liegt die Impfrate in Schweizer Hausarztpraxen bei knapp 2\%. Eine Studie sucht Gründe.
\end{abstract}

Posted at the Zurich Open Repository and Archive, University of Zurich ZORA URL: https://doi.org/10.5167/uzh-98046

Journal Article

Originally published at:

Djalali, S; Badertscher, N (2014). Impfung gegen Pneumokokken überzeugt nicht restlos. PrimaryCare, 14(13):210 


\section{Impfung gegen Pneumokokken überzeugt nicht restlos}

\begin{abstract}
Die Pneumokokkenimpfung gehört nicht zu den «beliebtesten" Impfungen. Bis vor kurzem empfahl das Bundesamt für Gesundheit, Risikopatienten jeden Alters und Menschen über 64 Jahre generell zu impfen. Dennoch liegt die Impfrate in Schweizer Hausarztpraxen bei knapp 2\%. Eine Studie sucht Gründe.
\end{abstract}

Zur Pneumokokkenimpfung existiert eine Reihe von klinischen und epidemiologischen Studien, eine qualitative Studie wurde erst jetzt durchgeführt. Im Mittelpunkt dieser Interviewstudie stand die Einstellung von Hausärzten zur Pneumokkokenimpfung. In der Tat sind Hausarztpraxen nach wie vor der Hauptort im Schweizer Gesundheitssystem an dem Impfungen verabreicht werden und entscheidend dafür, dass Impfungen die Bevölkerung erreichen können.

\section{Niedrige Impfrate}

Bis Anfang 2014 empfahl das Bundesamt für Gesundheit, alle Menschen über 64 Jahre und Risikopatienten generell gegen Pneumokokken zu impfen. Statistiken zeigen, dass jedoch nur knapp $2 \%$ der Zielgruppe erreicht wurden. In den semistrukturierten Studieninterviews mit 20 HausärztInnen, wurden mögliche Barrieren für die Pneumokkokenimpfung in der Hausarztpraxis diskutiert.

\section{Niedrigere Priorität}

Die Kolleginnen und Kollegen, die an der Studie teilnahmen, repräsentierten sowohl Hausarztpraxen in ländlichen, städtischen und Agglomerationsgebieten der Deutschschweiz, waren im Schnitt 55 Jahre alt und hatten 20 Jahre Berufserfahrung. Die Impfempfehlung war allen bekannt, wurde aber nicht als obligatorisch wahrgenommen und einer niedrigeren Priorität zugeordnet als andere Impfungen etwa die Influenzaimpfung.

\section{Unsichtbare Wirkung}

Die Ergebnisse zeigten, dass die Pneumokokkenimpfung im Praxisalltag untergeht, weil viele andere Belange die Konsultationen dominieren und verifizierte Pneumokokkeninfekte in der Praxis auch rar sind, weil bei respiratorischen Infekten in der Regel keine Abstriche genommen werden und empirisch behandelt wird. Einige Studienteilnehmer gaben sogar an, in ihrer gesamten Praxislaufbahn noch keine Pneumokokkeninfektion gesehen zu haben.

Weil die Infekte nicht gesehen resp. Pneumokokken zugeordnet werden, lässt sich für Hausärzte empirisch auch nicht abschätzen, welche Infekte durch die Impfung hätten vermieden werden können.

\section{Kontroverse Literatur}

Die Antworten der Studienteilnehmer spiegelten wider, dass die Effektivität der Impfung von den Hausärzten empirisch kaum erfasst werde. Dies könnte durch Literaturrecherchen und Weiterbildungen ausgeglichen werden, doch hier berichteten die Kollegen, dass die aktuelle Datenlage in der Literatur und die kontrovers ge- führten Expertendiskussionen sie nicht vorbehaltlos vom positiven Nutzen der Pneumokokkenimpfung überzeugen könnten.

\section{Ins Gespräch kommen}

Wohl auch deshalb werde die Impfung von Hausärzten selten proaktiv an die Patienten herangetragen. Die Patienten selbst fragten laut den Studienteilnehmern selten, die Vermutung wurde geäussert, dass die wenigsten Patienten überhaupt Bescheid wüssten, dass die Impfung existiere.

Um dies zu ändern, schlugen die Teilnehmer unter anderem vor, kombinierte Impfkampagnen für Influenza und Pneumokokken zu lancieren. Vor allem aber wünschten sich die Kollegen mehr gute Studien mit harten Endpunkten und epidemiologischen Kennzahlen wie «number needed to vaccine» (Anzahl von geimpften Menschen, die nötig ist um einen Infekt zu verhindern) und «number needed to harm» (Anzahl von Menschen, die geimpft wird bis ein Fall von Nebenwirkungen auftritt), die ihnen eine klare Meinungsbildung zum Thema und Positionsvertretung gegenüber den Patienten erlauben würden.

Zwischenzeitlich haben sich die Empfehlungen des BAG geändert. Die Impfung für Erwachsene wird nur noch für Risikopatienten unabhängig vom Alter - empfohlen. Grund für den Entscheid waren die kontroverse Datenlage und der fragliche epidemiologische Nutzen.

Literatur

- Badertscher N, Morell S, Rosemann T, Tandjung R. General practitioners' experiences, attitudes, and opinions regarding the pneumococcal vaccination for adults: a qualitative study. Int J Gen Med. 2012;5:967-974.

\section{Korrespondenz:}

Dr. med. Sima Djalali

Wissenschaftliche Mitarbeiterin

Institut für Hausarztmedizin Universität Zürich

Pestalozzistrasse 24, 8091 Zürich

sima.djalali[at]usz.ch, www.hausarztmedizin.uzh.ch

\section{PrimaryResearch - das Fenster zur Forschung}

In dieser Artikelserie stellen wir Forschungsarbeiten aus dem Institut für Hausarztmedizin der Universität Zürich (IHAMZ) vor. Die Originalarbeiten sind entweder «open access» zugänglich oder beim jeweiligen Autor auf Anfrage erhältlich. Die Ergebnisse geben einen spannenden Einblick in die täglichen Herausforderungen, aber auch die Leistung der Hausarztmedizin. An dieser Stelle ein herzlicher Dank an alle Kolleginnen und Kollegen, die sich an den Projekten beteiligen und die hier präsentierten Ergebnisse erst ermöglicht haben!

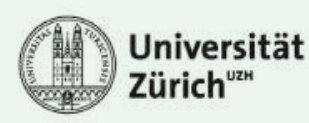

Institut für Hausarztmedizin 